\title{
Sensing and atomic-scale structure analysis of single nuclear-spin clusters in diamond
}

\author{
Fazhan Shi ${ }^{1}$, Xi Kong ${ }^{1}$, Pengfei Wang ${ }^{1}$, Fei Kong ${ }^{1}$, Nan Zhao ${ }^{2}$, Ren-Bao Liu ${ }^{3}$ and Jiangfeng Du ${ }^{1 \star}$
}

Single-molecule nuclear magnetic resonance is a current challenge in the field of magnetic resonance spectroscopy and has important applications in chemical analysis and quantum computing. Through decoherence measurements of nitrogenvacancy centres under dynamical decoupling control, the sensing of a single ${ }^{13} \mathrm{C}$ nuclear spin at nanometre distance has recently been realized $^{1-3}$. A further step towards the ultimate goal of structure analysis of single molecules would be the direct measurement of the interactions within single nuclearspin clusters ${ }^{4}$. Here we sense a single ${ }^{13} \mathrm{C}-{ }^{13} \mathrm{C}$ nuclear-spin dimer located about $1 \mathrm{~nm}$ from the nitrogen-vacancy centre and characterize the interaction $(\sim 690 \mathrm{~Hz})$ between the two nuclear spins. From the measured interaction we derive the spatial configuration of the dimer with atomic-scale resolution. These results indicate that, in combination with advanced material-surface engineering, central spin decoherence under dynamical decoupling control may be a useful probe for nuclear magnetic resonance single-molecule structure analysis.

Using nuclear magnetic resonance (NMR), the structures of complex molecules can be unravelled by analysis of the interaction between nuclear spins. Traditional magnetic resonance techniques rely on large ensembles of nuclear spins to accumulate a large signal-to-noise ratio. Recently, new methods, such as magnetic resonance force microscopy ${ }^{5}$ and optically detected magnetic resonance ${ }^{6,7}$, have been developed to improve sensitivity. Despite this progress, the ultimate goal is still single-molecule NMR. To achieve this goal, two important problems (sensing individual non-interacting nuclear spins and resolving interactions of nuclear-spin clusters) have to be solved. Non-interacting single nuclear spins have been successfully sensed using nitrogen-vacancy centre probes under dynamical decoupling control ${ }^{1-3}$. However, measurement of the interaction between nuclear spins within a single molecule and the resolution of their structure is still a challenge. Previous theoretical work ${ }^{4}$ provided a step towards this goal. Inspired by this theoretical precursor, we report here the first experimental characterization of coherent coupling within a two-nuclear-spin cluster close to a nitrogen-vacancy centre in diamond. The determination of the cluster's spatial configuration was made using the measured interaction.

The nitrogen-vacancy centre consists of a nitrogen impurity and a neighbouring vacancy. Its triplet $(S=1)$ ground state can be spin-polarized and read out optically. It has an ultralong coherence time of up to milliseconds in ultrapure samples ${ }^{8}$ or under dynamical decoupling control ${ }^{9}$. These remarkable properties make the nitrogen-vacancy centre a potential candidate for magnetic
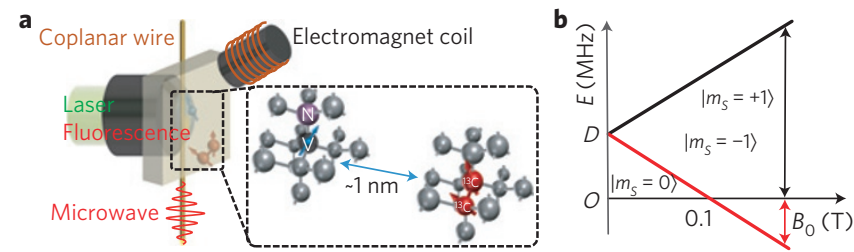

C

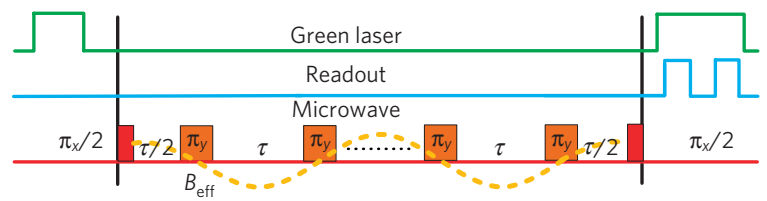

Figure 1 | Schematic of the set-up and experimental method. a, We measured the spin coherence of a nitrogen-vacancy sensor coupled to a ${ }^{13} \mathrm{C}-{ }^{13} \mathrm{C}$ dimer. The external d.c. magnetic field was applied by a movable electromagnet and microwaves were carried by a coplanar wire. b, Energy level scheme of the nitrogen-vacancy centre. The zero-field splitting value $D$ is $2,870 \mathrm{MHz}$. The eigenvalue of state $\left|m_{S}=-1\right\rangle\left(\left|m_{S}=+1\right\rangle\right)$, shown by the red (black) line, is linearly dependent on the external magnetic field $B_{0}$ We encoded the quantum transition $|0\rangle \leftrightarrow|-1\rangle$ (or $|0\rangle \leftrightarrow|+1\rangle$ ) as a qubit and manipulated it by resonant microwave pulses. c, The pulse sequences contained two green laser beam pulses for initialization and readout of the electron spin state with CPMG microwave pulses in between to control the spin. Microwave pulses $\pi_{x} / 2$ shown in red are the initial and readout $90^{\circ}$ pulses about the $x$ axis, and $\pi_{y}$ pulses are $180^{\circ}$ refocusing operations along the $y$ axis.

field sensing ${ }^{1-3,6,7,10-13}$, observing new quantum phenomena ${ }^{14,15}$ and quantum information processing ${ }^{16-24}$.

In pure diamond samples, nitrogen-vacancy centre electron spins lose their coherence owing to the magnetic fluctuations from surrounding ${ }^{13} \mathrm{C}$ nuclear spins. The fluctuations are dominated by single nuclear-spin precession or flip-flop processes of spin clusters. Our measurement was carried out in a strong fields, where single spin precession was strongly suppressed ${ }^{25}$ and therefore the interacting ${ }^{13} \mathrm{C}$ spin clusters were the dominant noise source. In this case, we are able to single out the physical effect caused by collective dynamics of nuclear-spin clusters and, then, unravel the interaction within the cluster.

Among the different kinds of ${ }^{13} \mathrm{C}$ nuclear-spin cluster, a bonded ${ }^{13} \mathrm{C}$ spin dimer stands out because it can induce strong fingerprint modulations on nitrogen-vacancy centre spin coherence ${ }^{4}$. In this

\footnotetext{
${ }^{1}$ National Laboratory for Physics Sciences at the Microscale and Department of Modern Physics, University of Science and Technology of China, Hefei 230026, China, ${ }^{2}$ Beijing Computational Science Research Center, Beijing 100084, China, ${ }^{3}$ Department of Physics and Centre for Quantum Coherence, The

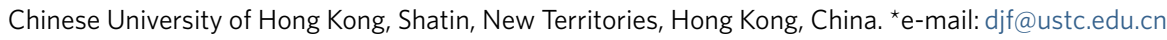



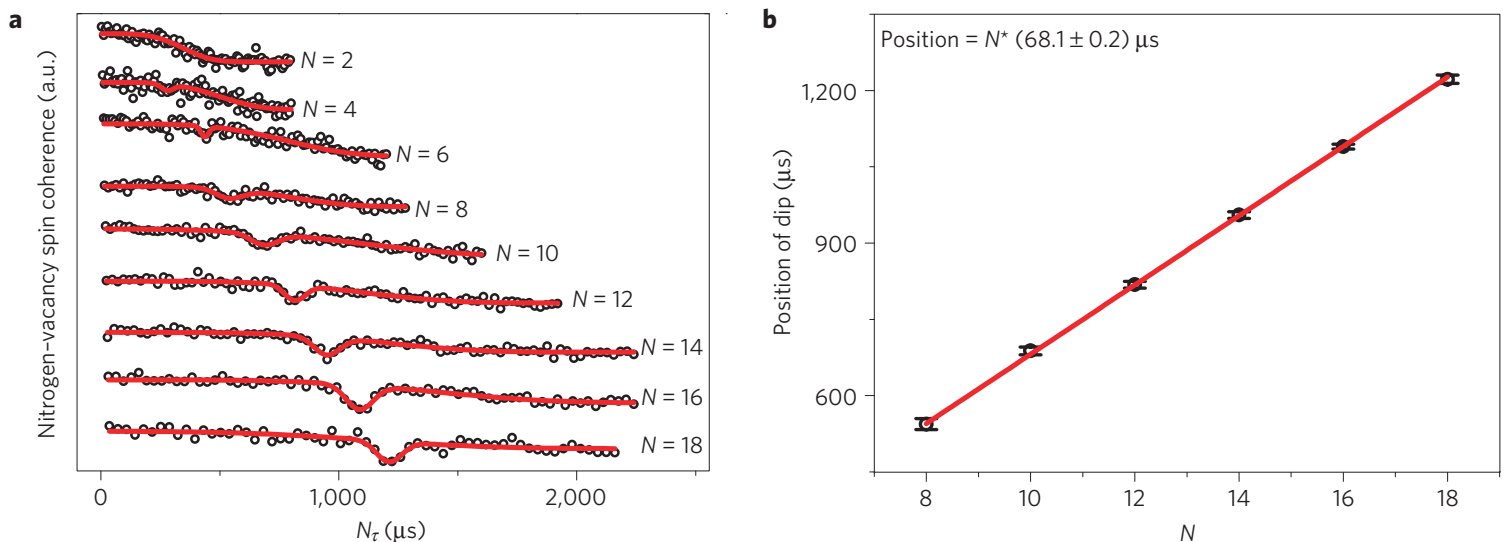

Figure 2 | Dip features in the nitrogen-vacancy centre spin decoherence under CPMG control with various numbers of pulses. a, As the pulse number $N$ of CPMG increased from 2 (top curve) to 18 (bottom curve), the dips induced by a spin dimer emerged and became more and more pronounced. The symbols are measured data and the curves are fits to $\left\{y_{0}+A_{0} \exp \left[-\left(t / T_{2}\right)^{4}\right]\right\} \times\left\{y_{1}+A_{1} \exp \left[-2\left(\left(t-t_{c}\right) / \omega\right)^{2}\right] / \omega / \sqrt{\pi / 2}\right\}$, which consists of a smooth profile and a Gaussian dip. $\mathbf{b}$, The positions of the dips are proportional $N$ in CPMG control and the red line is a fit to the data given by $N(68.1 \pm 0.2) \mu s$. The vertical error bars indicate the uncertainty of the fit of the measured spin coherence data in $\mathbf{a}$
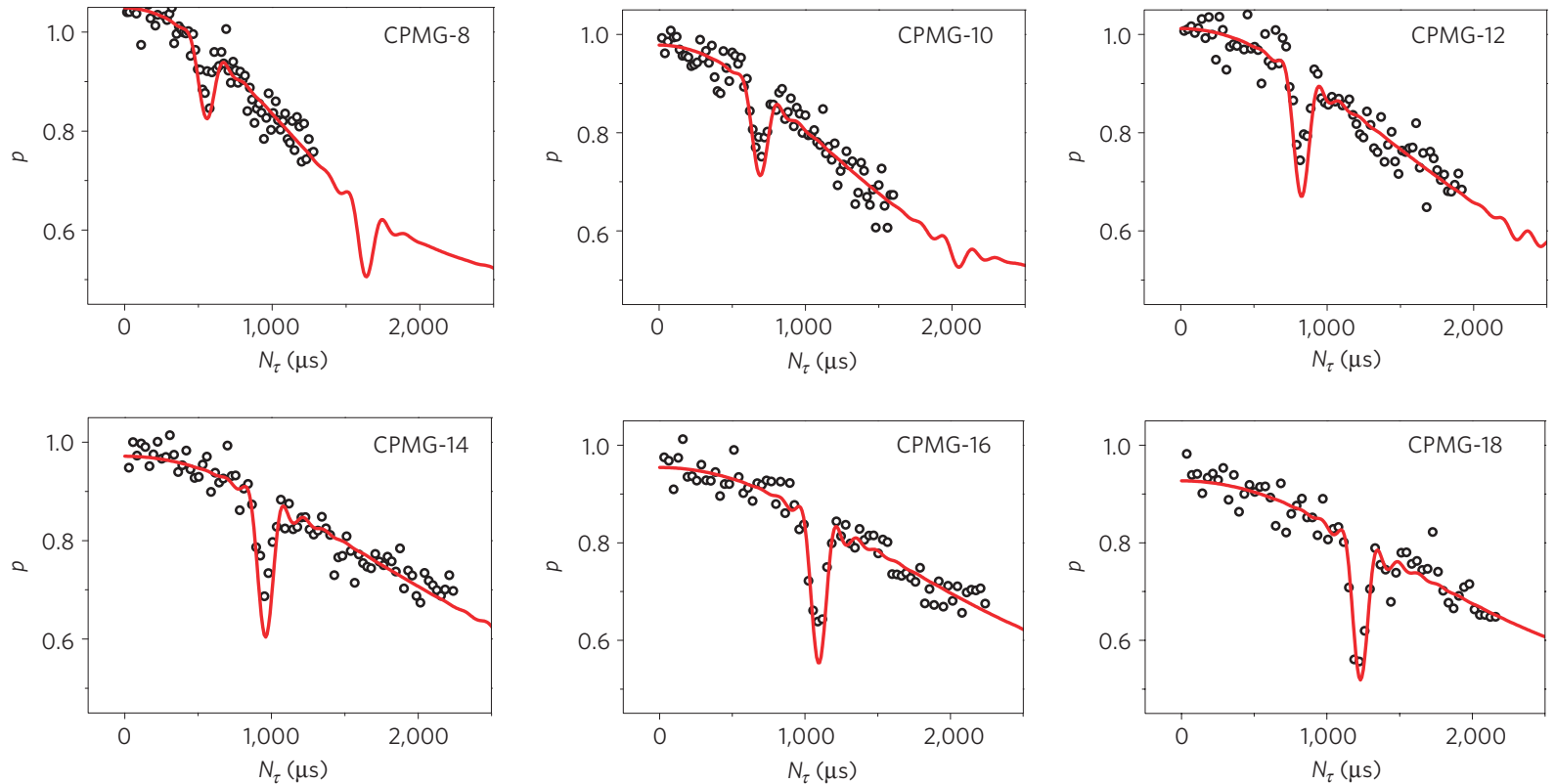

Figure 3 | Characterizing the interaction of a nuclear-spin dimer by fingerprint-matching between measurement and simulation. Nitrogen-vacancy spin coherence as a function of total evolution time under CPMG dynamical decoupling control with various numbers of pulses $(N=8,10,12,14,16$ and 18$)$. The experimental data (symbols) are well reproduced by simulations (lines) based on a dimer with interaction $\left[X, Z^{\left(m_{\mathrm{s}}\right)}\right]=[0.685,14.6] \mathrm{kHz}$. Other nuclear-spin pairs do not produce dip features matching the experimental data. In these measurements the magnetic field is $B_{0}=1,770 \mathrm{G}$. The vertical axes show the probability in the $|0\rangle$ state.

work, we observed the dimer-induced modulation on the nitrogenvacancy centre electron spin coherence, and characterized the interaction both within the dimer and between the dimer and the nitrogen-vacancy centre electron spin.

In a strong magnetic field, the dimer dynamics can be described by a pseudo-spin model, where the two-spin states $|\uparrow \downarrow\rangle$ and $|\downarrow \uparrow\rangle$ of the dimer are mapped to the spin-up $|\uparrow\rangle$ and spin-down $|\downarrow\rangle$ states of the pseudo-spin $\boldsymbol{\sigma}$. The polarized dimer states $|\uparrow \uparrow\rangle$ and $|\downarrow \downarrow\rangle$ are energetically separated and irrelevant in the dynamics because of the large Zeeman energy in the strong field. The dynamics of the pseudo-spin is governed by the Hamiltonian

$$
H_{\mathrm{ps}}^{\left(m_{s}\right)}=\frac{1}{2} \mathbf{h}^{\left(m_{s}\right)} \cdot \boldsymbol{\sigma}=\frac{1}{2}\left(X \sigma_{x}+Z^{\left(m_{s}\right)} \sigma_{z}\right)
$$

where the component $X$ of the pseudo-field $\mathbf{h}^{(\alpha)}$ is the nuclear dipolar interaction strength and the component $Z^{\left(m_{s}\right)}$ is due to the hyperfine couplings difference of the two nuclei induced by the nitrogen-vacancy centre electron spin state $\left|m_{S}\right\rangle$.

The pseudo-field component $Z^{\left(m_{s}\right)}$ and hence the nuclear-spin pair dynamics depend on the nitrogen-vacancy centre electron spin state $\left|m_{S}\right\rangle$. For the nitrogen-vacancy centre spin in the $\left|m_{S}=0\right\rangle$ state, the hyperfine splitting vanishes, whereas for the $\left|m_{S}=1\right\rangle$ state, the hyperfine splittings of the two nuclear spins have a finite different value of $Z^{(1)} \sim 10 \mathrm{kHz}$. Correspondingly, the pseudo-spin will precess with frequencies $\omega_{\mathrm{ps}}^{(0)}=X$ or $\omega_{\mathrm{ps}}^{(1)}=\sqrt{X^{2}+\left[Z^{(1)}\right]^{2}}$. When the nitrogen-vacancy centre spin is prepared in a superposition state $\left(\left|m_{S}=0\right\rangle+\left|m_{S}=1\right\rangle\right) / \sqrt{2}$, the characteristic frequency of the noise induced by the pseudo-spin becomes $\omega_{\mathrm{ps}}^{\text {eff }}=\sqrt{X^{2}+\left[Z^{(1)} / 2\right]^{2}}$ 
a

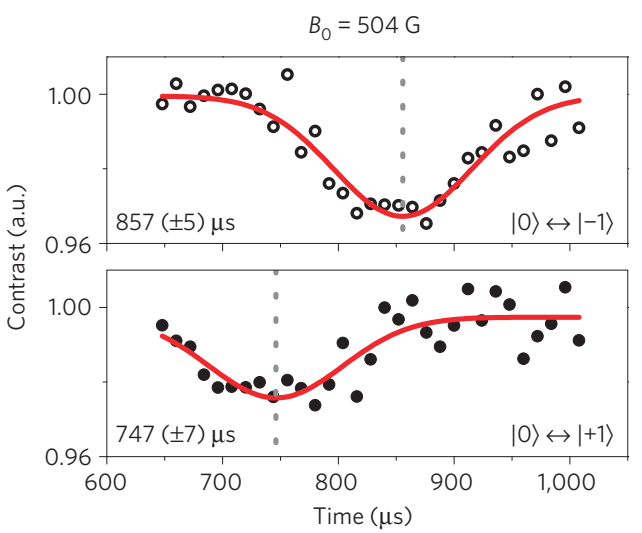

C
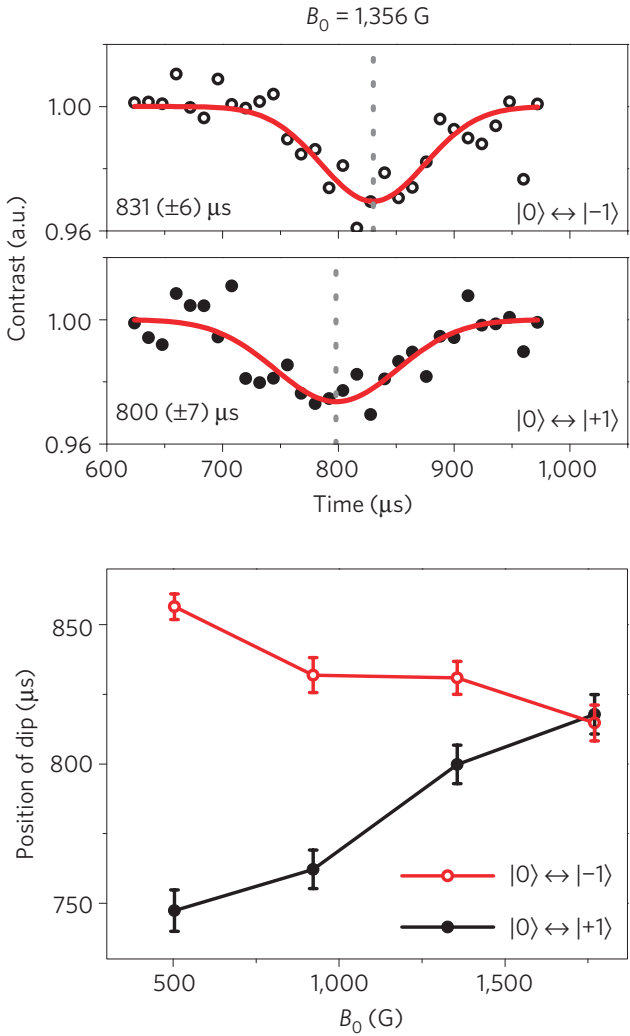

b

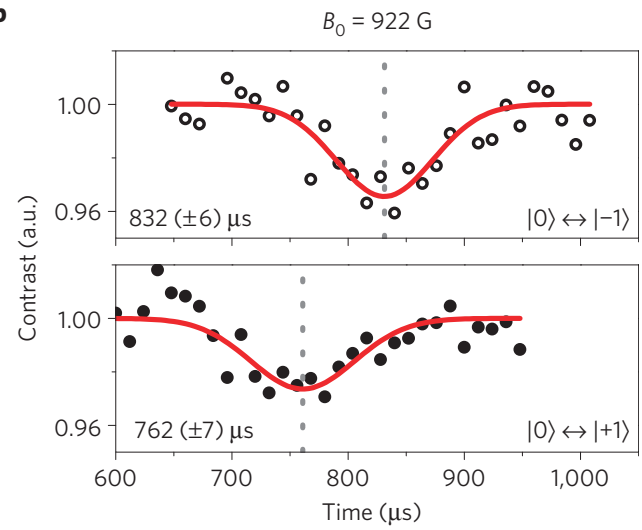

d

d

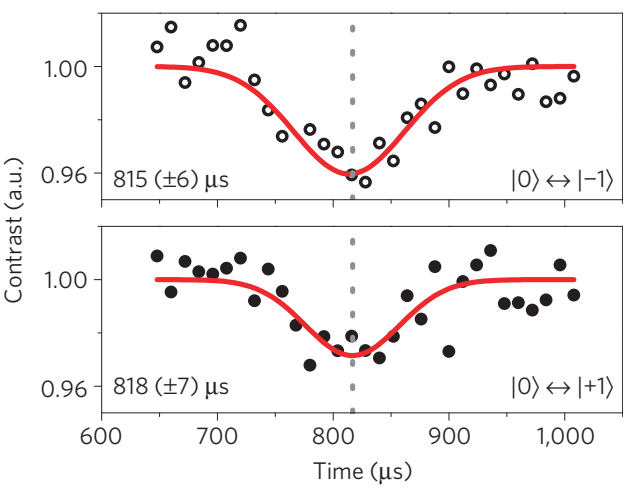

f

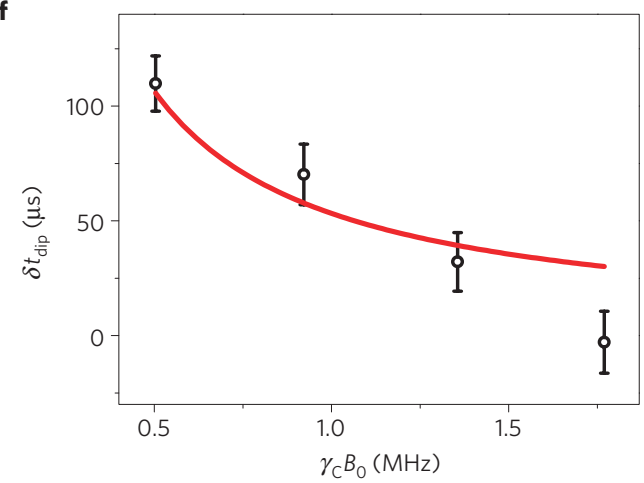

Figure 4 | Magnetic field dependence of the dip features in the nitrogen-vacancy centre spin decoherence for different transitions. a-d, The upper and lower panels in each part show the CPMG-12 decoherence of the transitions $|0\rangle \leftrightarrow|+1\rangle$ and $|0\rangle \leftrightarrow|-1\rangle$, respectively. The magnetic field for a-d was, respectively, 504, 922, 1,356 and 1,770 G. e, Field dependence of the dip positions for different nitrogen-vacancy centre spin transitions. The error bars indicate the uncertainty of the fit of the measured data in a-d. f, Comparison between the measured (symbols) and calculated (lines) positions of the dips as functions of the magnetic field. The error bar of each point in $\mathbf{f}$ is obtained from the uncertainty of the corresponding points in $\mathbf{e}$ according to the error propagation formula.

(see Supplementary Section 1.2). In our experiment, the pseudospin precession frequency $\omega_{\mathrm{ps}}^{\text {eff }}$ was measured by dynamical decoupling control ${ }^{9,26,27}$ of the nitrogen-vacancy centre spin.

The set-up and experimental scheme are schematically shown in Fig. 1. The periodic Carr-Purcell-Meiboom-Gill (CPMG) dynamical decoupling control sequences are employed to realize 'quantum lock-in detection'28, which enhances sensitivity to all fields oscillating synchronously with the pulse spacing while suppressing the effects of field fluctuations at every other frequency. In the intuitive picture (Fig. 1c), the transition in the ${ }^{13} \mathrm{C}$ dimer imposes an a.c. noise with a characteristic frequency. Therefore, when the CPMG timing matches the noise characteristic frequency, the decoupling fails markedly and there is a sharp dip in the nitrogen-vacancy spin coherence ${ }^{3,29}$. This allows one to identify the characteristic precession frequency. Figure 2 shows the nitrogenvacancy centre spin coherence under CPMG- $N$ control. For pulse number $N>4$, we observed coherence dips on top of a smooth decoherence. The dip position $t_{\text {dip }}$ is proportional to the pulse number $N$ (Fig. 2b). As shown in previous work ${ }^{3,4}$, the dip position is related to the precession frequency by

$$
t_{\mathrm{dip}}=\frac{N \pi}{\omega_{\mathrm{ps}}^{\mathrm{eff}}}
$$

from which we extract the pseudo-spin precession frequency $\omega_{\mathrm{ps}}^{\mathrm{eff}} \approx 2 \pi \times(7.3 \pm 0.1) \mathrm{kHz}$.

The pseudo-field components $X$ and $Z^{\left(m_{s}\right)}$ were determined by comparing the observed dip widths and depths under various 
control pulse numbers $N$ with the calculated electron spin coherence using the pseudo-spin model. As the nuclear-spin positions are discrete on diamond lattice, the dipolar interaction takes discrete values $X=2.1 \mathrm{kHz}$ for a dimer in the [111] direction, $X=0.685 \mathrm{kHz}$ for a dimer in other $\mathrm{C}-\mathrm{C}$ bond directions, and $X<0.3 \mathrm{kHz}$ for nuclear spins at a larger distance. The calculation shows that only the parameters $\left[X, Z^{(1)}\right]=[0.685,14.6] \mathrm{kHz}$ yield full agreement with the experiment data shown in Fig. 3. With this we conclude that the coherence dip was caused by a dimer that was not orientated along the [111] direction (see Supplementary Section 3.1).

We characterized the coupling between the dimer and the nitrogen-vacancy centre electron spin from measurement under various magnetic fields. The $Z^{\left(m_{s}\right)}$ component of the pseudo-field consists of two parts $Z^{\left(m_{s}\right)}=Z_{\|}^{\left(m_{s}\right)}+Z_{\perp}^{\left(m_{s}\right)}\left(B_{0}\right)$. The parallel part $Z_{\|}^{\left(m_{s}\right)}=A_{1, \|}-A_{2, \|}$ is the difference of the hyperfine fields projected to the applied magnetic field direction. The transverse part $Z_{\perp}^{\left(m_{s}\right)}\left(B_{0}\right)=\left(A_{1, \perp}^{\left(m_{S}\right)}\right)^{2}-\left(A_{2, \perp}^{\left(m_{s}\right)}\right)^{2} / \gamma_{C} B_{0}$ comes from the perturbative frequency shifts of the two spins induced by virtual flipping through the polarized states $|\uparrow \uparrow\rangle$ and $|\downarrow \downarrow\rangle$ (ref. 25), where $\gamma_{C}$ is the gyromagnetic ratio of ${ }^{13} \mathrm{C}$ nuclear spin and $\gamma_{\mathrm{C}} B_{0}$ is the Larmor precession frequency. Note that the parallel part is opposite for $\left|m_{S}=1\right\rangle$ and $\left|m_{S}=-1\right\rangle$ states, but the transverse part is the same. The presence of the transverse part $Z_{\perp}^{\left(m_{S}\right)}\left(B_{0}\right)$ causes different dip positions for the transitions $\left|m_{S}=0\right\rangle \leftrightarrow|+1\rangle$ and $\left|m_{S}=0\right\rangle \leftrightarrow|-1\rangle$ (Fig. 4a-e). The dip position difference $\delta t_{\text {dip }}$ depends on the magnetic field (Fig. 4f). In the case of $Z_{\|}^{\left(m_{s}\right)} \gg Z_{\perp}^{\left(m_{S}\right)}\left(B_{0}\right)$, which is the case in our experiment,

$$
\delta t_{\text {dip }} \approx \frac{2 N}{\left(Z_{\|}^{(1)}\right)^{2}} \frac{\left(A_{1, \perp}\right)^{2}-\left(A_{2, \perp}\right)^{2}}{\gamma_{\mathrm{C}} B_{0}}
$$

In the strong magnetic field limit, $\delta t_{\text {dip }} \rightarrow 0$, and $Z_{\|}^{(1)}=14.6 \mathrm{kHz}$ is extracted from $\omega_{\mathrm{ps}}^{\text {eff }}$, as shown in Fig. 3 for $B=1,770 \mathrm{G}$. The magnetic field dependence of $\delta t_{\text {dip }}$ gives the difference of the transverse hyperfine coupling $\delta A_{\perp}^{2}=\left(A_{1, \perp}\right)^{2}-\left(A_{2, \perp}\right)^{2}=(21.7 \mathrm{kHz})^{2}$; see Supplementary Section 3.2). From the values of $Z^{(1)}$ and $\delta A_{\perp}^{2}$, we determined that the dimer was located about $1 \mathrm{~nm}$ away from the nitrogen-vacancy centre (see Supplementary Section 4). The field dependence of the dip features also excludes electron spin clusters as the cause of the coherent modulation (see Supplementary Section 5).

In summary, we have observed and characterized a nuclearspin interaction as weak as $685 \mathrm{~Hz}$ within a single two-nuclearspin cluster in diamond. From decoherence measurements of the nitrogen-vacancy centre spin under multi-pulse dynamical decoupling control and various magnetic fields, we determine, with atomic-scale resolution, the orientation and separation of the two nuclear spins, and their distance from the nitrogen-vacancy centre. These results demonstrate that the dynamical decoupling technique can be used for detecting the interaction between nuclear spins. Recent investigations ${ }^{30}$ have shown that, with well-designed preparation conditions, shallow nitrogen-vacancy centres with distances from the diamond surface of several nanometres could have similar coherence times to nitrogen-vacancy centres in bulk; hence, shallow nitrogen-vacancy centres can be used for structure analysis of single molecules located on the diamond surface. With this observation, this work provides a first proof-of-principle demonstration of the detection of interaction between nuclear spins and could be the first step towards the detection of interaction inside single molecules. This work, together with the previous work on individual nuclear spins ${ }^{1-3}$, opens possibilities for the field of single molecule spin resonance spectroscopy (see Supplementary Section 1.3). Nonetheless, it has to be noted that a great deal of challenges in materials engineering and control are to be overcome to extend the structure analysis of a nuclear-spin cluster embedded in the lattice to the ultimate goal of NMR of single molecules on a surface.

\section{Methods}

The nitrogen-vacancy sensor in [100] face bulk diamond was mounted on a typical optically detected magnetic resonance confocal set-up that was synchronized together with the microwave bridge by a multichannel pulse generator (Spincore, PBESR-PRO-500). The nitrogen concentration was less than $5 \mathrm{ppb}$ and the abundance of ${ }^{13} \mathrm{C}$ was at the natural level of $1.1 \%$. The pump $532 \mathrm{~nm}$ green laser beam and phonon sideband fluorescence (wavelength, 650-800 nm) went through the same oil objective (Olympus, PLAPON $60^{\star} \mathrm{O}$, NA 1.42). To protect the nitrogen-vacancy sensor $T_{1}$ time from laser leakage effects the pump beam was passed twice through an acousto-optic modulator (ISOMET, power leakage ratio $\sim 1 / 1,000$ ) before it went into the objective. The fluorescence intensity, collected by avalanche photodiodes (Perkin Elmer, SPCM-AQRH-14) with a counter card, was used to monitor the magnetic signal on the nitrogen-vacancy sensor. The adjustable external magnetic field $(0-2,000 \mathrm{G})$, created by the electrical coil (GMW model 5201, $3 \mathrm{ppm} \mathrm{h}^{-1}$ stability), was aligned by monitoring the variation of fluorescence counts (see Supplementary Section 2.1). Pulsed microwaves were carried by a $20 \mu \mathrm{m}$ copper wire. The microwave phase was continuously adjusted by a mechanical phase shifter and was fixed to the $X$ and $Y$ phases by a network analyser (Agilent). High-order CPMG- $N$ sequences were used to amplify the weak signal $Z^{\left(m_{s}\right)}$ from the remote nuclear-spin pair, $Z^{\left(m_{s}\right)}-\mathrm{kHz} \ll \mathrm{MHz} \sim 1 / T_{2}^{*}$, where, $T_{2}^{*}$ was roughly $1 \mu$ s in our sample. CPMG-12 on the two transitions $|0\rangle \leftrightarrow| \pm 1\rangle$ under various magnetic fields was employed to characterize the coupling vector and distinguish the high-field threshold. On the basis of the experimental conditions, the pseudo-spin model was used to analyse the results. In summary, we have devised a method for resolving the structure and location of a nuclear-spin dimer (see Supplementary Section 6).

Received 8 May 2013; accepted 18 October 2013; published online 24 November 2013

\section{References}

1. Kolkowitz, S., Unterreithmeier, Q. P., Bennett, S. D. \& Lukin, M. D. Sensing distant nuclear spins with a single electron spin. Phys. Rev. Lett. 109, 137601 (2012).

2. Taminiau, T. H. et al. Detection and control of individual nuclear spins using a weakly coupled electron spin. Phys. Rev. Lett. 109, 137602 (2012).

3. Zhao, N. et al. Sensing single remote nuclear spins. Nature Nanotech. 7, 657-662 (2012).

4. Zhao, N., Hu, J. L., Ho, S. W., Wan, J. T. K. \& Liu, R. B. Atomic-scale magnetometry of distant nuclear spin clusters via nitrogen-vacancy spin in diamond. Nature Nanotech. 6, 242-246 (2011).

5. Degen, C. L., Poggio, M., Mamin, H. J., Rettner, C. T. \& Rugar, D. Nanoscale magnetic resonance imaging. Proc. Natl Acad. Sci. USA 106, 1313-1317 (2009).

6. Balasubramanian, G. et al. Nanoscale imaging magnetometry with diamond spins under ambient conditions. Nature 455, 648-651 (2008).

7. Maze, J. R. et al. Nanoscale magnetic sensing with an individual electronic spin in diamond. Nature 455, 644-647 (2008).

8. Balasubramanian, G. et al. Ultralong spin coherence time in isotopically engineered diamond. Nature Mater. 8, 383-387 (2009).

9. Ryan, C. A., Hodges, J. S. \& Cory, D. G. Robust decoupling techniques to extend quantum coherence in diamond. Phys. Rev. Lett. 105, 200402 (2010).

10. Staudacher, T. et al. Nuclear magnetic resonance spectroscopy on a (5-nanometre) ${ }^{3}$ sample volume. Science 339, 561-563 (2013).

11. Mamin, H. J. et al. Nanoscale nuclear magnetic resonance with a nitrogen-vacancy spin sensor. Science 339, 557-560 (2013).

12. Maletinsky, P. et al. A robust scanning diamond sensor for nanoscale imaging with single nitrogen-vacancy centres. Nature Nanotech. 7, 320-324 (2012).

13. Loretz, M. et al. Radio-frequency magnetometry using a single electron spin. Phys. Rev. Lett. 110, 017602 (2013).

14. Huang, P. et al. Observation of an anomalous decoherence effect in a quantum bath at room temperature. Nature Commun. 2, 570 (2011).

15. Zhao, N., Wang, Z. \& Liu, R. B. Anomalous decoherence effect in a quantum bath. Phys. Rev. Lett. 106, 217205 (2011).

16. Dutt, M. V. G. et al. Quantum register based on individual electronic and nuclear spin qubits in diamond. Science 316, 1312-1316 (2007).

17. Neumann, P. et al. Multipartite entanglement among single spins in diamond. Science 320, 1326-1329 (2008).

18. Fuchs, G. D. et al. Gigahertz dynamics of a strongly driven single quantum spin. Science 326, 1520-1522 (2009).

19. Cappellaro, P., Jiang, L., Hodges, J. S. \& Lukin, M. D. Coherence and control of quantum registers based on electronic spin in a nuclear spin bath. Phys. Rev. Lett. 102, 210502 (2009). 
20. Neumann, P. et al. Quantum register based on coupled electron spins in a room-temperature solid. Nature Phys. 6, 249-253 (2010).

21. Shi, F. Z. et al. Room-temperature implementation of the Deutsch-Jozsa algorithm with a single electronic spin in diamond. Phys. Rev. Lett. 105, 040504 (2010).

22. Xu, X. K. et al. Coherence-protected quantum gate by continuous dynamical decoupling in diamond. Phys. Rev. Lett. 109, 070502 (2012).

23. Sar, T. V. D. et al. Decoherence-protected quantum gates for a hybrid solid-state spin register. Nature 484, 82-86 (2012).

24. Dolde, F. et al. Room-temperature entanglement between single defect spins in diamond. Nature Phys. 9, 139-143 (2013).

25. Zhao, N., Ho, S. W. \& Liu, R. B. Decoherence and dynamical decoupling control of nitrogen vacancy centre electron spins in nuclear spin baths. Phys. Rev. B 85, 115303 (2012).

26. Du, J. F. et al. Preserving electron spin coherence in solids by optimal dynamical decoupling. Nature 461, 1265-1268 (2009).

27. de Lange, G., Wang, Z. H., Riste, D., Dobrovitski, V. V. \& Hanson, R. Universal dynamical decoupling of a single solid-state spin from a spin bath. Science 330, 60-63 (2010)

28. Kotler, S., Akerman, N., Glickman, Y., Keselman, A. \& Ozeri, R. Single-ion quantum lock-in amplifier. Nature 473, 61-65 (2011).

29. Lange, G. de., Riste, D., Dobrovitski, V. V. \& Hanson, R. Single-spin magnetometry with multipulse sensing sequences. Phys. Rev. Lett. 106, $080802(2011)$.

30. Ofori-Okai, B. K. et al. Spin properties of very shallow nitrogen vacancy defects in diamond. Phys. Rev. B 86, 081406(R) (2012).

\section{Acknowledgements}

This work was supported by the National Key Basic Research Program of China (Grant No. 2013CB921800), the National Natural Science Foundation of China (Grant Nos. $11227901,91021005,10834005,11028510)$, the 'Strategic Priority Research Program (B)' of the CAS (Grant No. XDB01030400), the Fundamental Research Funds for the Central Universities, Hong Kong Research Grants Council (N_CUHK403/11, 402410 \& HKU8/CRF/11G), the Chinese University of Hong Kong Focused Investments Scheme and the 1000 Plan Program for Young Talents. The authors thank A. Crosse for help to improve the readability of the manuscript.

\section{Author contributions}

J.D. and R-B.L. proposed the idea. J.D. and F.S. designed the experimental proposal. P.W., X.K. and F.S. prepared the experimental set-up. F.S., F.K. and X.K. performed the experiments. J.D. supervised the set-up and experiments. N.Z. and F.S. carried out the calculation. F.S., N.Z., R-B.L. and J.D. wrote the paper. All authors analysed the data, discussed the results and commented on the manuscript.

\section{Additional information}

Supplementary information is available in the online version of the paper. Reprints and permissions information is available online at www.nature.com/reprints. Correspondence and requests for materials should be addressed to J.D.

\section{Competing financial interests}

The authors declare no competing financial interests. 